\title{
A machine vision approach for detecting and inspecting circular parts
}

\author{
Du-Ming Tsai \\ Machine Vision Lab. \\ Department of Industrial Engineering and Management \\ Yuan-Ze University, Chung-Li, Taiwan, R.O.C. \\ E-mail: iedmtsai@ saturn.yzu.edu.tw
}

(Figures are not available in this report)

\begin{abstract}
In this paper, we present a machine vision approach for detecting and inspecting circular parts and the parts with circular arcs on the contours. The method uses the Hough transform technique and utilizes the directional information of a normal to the circle at each boundary point. A cubic polynomial curve fitting is used to estimate the normal and determine the concavity of the fitted curve at each given boundary point. The proposed Hough transform method is a two-stage procedure. The first stage of the procedure uses a 2-D accumulator array to detect circle centers. Then the second stage uses a 1-D accumulator array to detect the radii of circles. The proposed method is robust to detect circular parts with partial occlusion such as peripheral defects or burrs. For an image of size $\mathrm{N} x \mathrm{~N}$, the storage requirements are $\mathrm{N}^{2}$ and the time complexity is bounded by $(\mathrm{N}+\mathrm{m}) \mathrm{n}$, where $\mathrm{m}$ is the number of circle centers detected in the first stage and $\mathrm{n}$ is the number of boundary points in the image.
\end{abstract}

Keywords: Machine vision, Circle detection, Part inspection, Hough transform 


\section{INTRODUCTION}

Detecting and locating circular parts from a digital image is important in industrial applications such as automatic inspection and robotic assembly. The radius and the center of a circle generally can be estimated by two distinct approaches: the least-squares curve fitting and the Hough transform.

Given a set of coordinates which represent the boundary of a part and presumably belong to a circular arc, the parameters of the circle can be estimated by minimizing the least mean square errors between the given boundary points and the curve $[1,2,3]$. For a complex part involving circular arcs and other non-circular segments on the contour, the boundary points must be grouped into meaningful circular segments before the use of least-squares fitting methods. In addition, this approach is very sensitive to noise and occlusions of parts.

The Hough transform (HT) techniques [4] for analytic shapes use a constraint equation to transform a set of feature points in image space into a set of accumulated votes in a parameter space. For each feature point, votes are accumulated in an accumulator array for all parameter combinations that satisfy the constraint equation. At the end of voting, those array elements containing large numbers of votes indicate the presence of the shape with the corresponding parameters. The HT converts a difficult global detection problem in image space into a more easily solved local peak detection problem in a parameter space. The primary benefits of using the HT is its robustness for noisy images and occluded parts. Since circles are completely defined by three parameters, namely the radius $r$ and the coordinates of the center $(\bar{x}, \bar{y})$, the conventional HT requires a three-dimensional array to detect circles in an image scene. To search for a circle whose center is within an image of size $\mathrm{Nx} \mathrm{N}$, and whose radius in not larger than $\mathrm{N}$ with 1 pixel 
resolution, would require a three-dimensional accumulator of size $\mathrm{N}^{3}$. This incurs considerable storage and computation.

The use of the HT to detect circles was first introduced by Duda and Hart [5]. An edge detection process is carried out to identify significant edge points. Then the positions of all possible center locations are accumulated in a parameter space for all anticipated radii. Conker [6] used the gradient orientations of two neighboring edge points to estimate the center of a circle. Since the center of a circle must lie along the gradient direction of each edge point on the circle, the intersection point of gradients is identified as the center. This approach can not discriminate concentric circles of different radii and is difficult to estimate the circle center to the required accuracy. Yip et al. [7] used parallel edge points to estimate the parameters of a circle and an ellipse. This approach only requires a two-dimensional accumulator, but will fail to detect the occluded circles on which pairs of parallel edge points are not presented simultaneously.

In this paper, we develop a fast two-stage Hough transform algorithm for detecting and inspecting partially occluded circular parts. The occlusion of parts may result from the peripheral breakdown, defects or burrs. The circular portions of parts such as cams also can be detected and measured using such algorithm. The proposed method consists of a two-dimensional accumulator to find circle centers followed by a one-dimensional accumulator to determine circle radii. The first stage begins with a boundary following process to find the sequence of boundary points of each part in the image. A cubic polynomial curve fitting method is employed to estimate the normal and the concavity of the fitted curve at each boundary point. Based on the normal direction and concavity information, the line segment that the circle center may lie on is determined. The votes are collected in a parameter plane based on the coordinates of each point on the resulting line segment. At the end of the first-stage voting process, those array elements containing large numbers of votes indicate the presence of circle centers. The second stage of the 
voting process calculates the radial distances between each center detected in the first stage and all boundary points in the image. The local peaks in the 1-D accumulator then indicate the presence of circle radii for a given circle center.

This paper is organized as follows: In section 2, the cubic polynomial curve fitting method for estimating the normal and the arc concavity at a boundary point is first introduced. Then the detection of circle centers followed by the detection of circle radii is discussed. In section 3 , experimental results are presented. The conclusion is given in section 4 .

\section{THE HT WITH NORMAL DIRECTIONS}

Let the normal to a curve at point $\mathrm{p}$ be the line passing through $\mathrm{p}$ and perpendicular to the tangent there. Since the center of a circle must lie along the normal direction of each boundary point on the circle, the common intersection point of these normals indicate the center of the circle. In this paper, we estimate best, in the least-squares sense, the slope of a normal by fitting a small segment of a digital curve in an image $b$ a continuous polynomial function. Since a small segment of circular arcs shown in the digital image may be represented by a straight line, especially the one in the horizontal or vertical direction, or by a curve involving an inflection point, a cubic polynomial function is employed to approximate the small segment. The image of scene parts is preprocessed by simple binary thresholding and boundary following [8] in order to extract the boundary points. Let the sequence of $\mathrm{n}$ digital points describe a boundary $\mathrm{P}$,

$$
P=\left\{p_{j}=\left(x_{j}, y_{j}\right), j=1,2, \ldots, n\right\}
$$

where $\mathrm{p}_{\mathrm{j}+1}$ is adjacent to $\mathrm{p}_{\mathrm{j}}$, and $\left(\mathrm{x}_{\mathrm{j}}, \mathrm{y}_{\mathrm{j}}\right)$ is the Cartesian coordinates of $\mathrm{p}_{\mathrm{j}}$ in the image. 
Let $p_{i}=\left(x_{i}, y_{i}\right)$ be the boundary point at which the normal is to be estimated. The region of support that defines the neighboring points of $\mathrm{p}_{\mathrm{i}}$ between points $\mathrm{p}_{\mathrm{i}-\mathrm{k}}$ and $\mathrm{p}_{\mathrm{i}+\mathrm{k}}$ for some integer $\mathrm{k}$ is given by

$$
N\left(p_{i}\right)=\left\{p_{j} \mid i-k \leq j \leq i+k\right\}
$$

Shift all points $\mathrm{p}_{\mathrm{j}}$ in $\mathrm{N}\left(\mathrm{p}_{\mathrm{i}}\right)$ by $\left(-\mathrm{x}_{\mathrm{i}},-\mathrm{y}_{\mathrm{i}}\right)$ and translate $\mathrm{p}_{1}$ to the origin of the new coordinate system. The size of the region of support k can be a predefined constant determined empirically, or it can be selected adaptively as proposed in [9]. The cubic polynomial function is represented by

$$
y=f(x)=a+b x+c x^{2}+d x^{3}
$$

where $a, b, c$ and $d$ are the four unknown coefficients to be estimated.

To minimize the accumulated distance errors between the observed points within the region of support and the curve, the objective function is given by

$$
\min F(a, b, c, d)=\sum_{j=i-k}^{i+k}\left[y_{j}-\left(a+b x_{j}+c x_{j}^{2}+d x_{j}^{3}\right)\right]^{2}
$$

Differentiating $\mathrm{F}(\mathrm{a}, \mathrm{b}, \mathrm{c}, \mathrm{d})$ with respect to $\mathrm{a}, \mathrm{b}, \mathrm{c}$ and $\mathrm{d}$, and setting them to zero, we obtain

$$
\mathrm{X}=\mathrm{A}^{-1} * \mathrm{~B}
$$

where

$$
X=[a, b, c, d]^{T}
$$




$$
\begin{aligned}
& \mathrm{A}=\left[\begin{array}{cccc}
\sum 1 & \sum \mathrm{x}_{\mathrm{j}} & \sum \mathrm{x}_{\mathrm{j}}^{2} & \sum \mathrm{x}_{\mathrm{j}}^{3} \\
\sum \mathrm{x}_{\mathrm{j}} & \sum \mathrm{x}_{\mathrm{j}}^{2} & \sum \mathrm{x}_{\mathrm{j}}^{3} & \sum \mathrm{x}_{\mathrm{j}}^{4} \\
\sum \mathrm{x}_{\mathrm{j}}^{2} & \sum \mathrm{x}_{\mathrm{j}}^{3} & \sum \mathrm{x}_{\mathrm{j}}^{4} & \sum \mathrm{x}_{\mathrm{j}}^{5} \\
\sum \mathrm{x}_{\mathrm{j}}^{3} & \sum \mathrm{x}_{\mathrm{j}}^{4} & \sum \mathrm{x}_{\mathrm{j}}^{5} & \sum \mathrm{x}_{\mathrm{j}}^{6}
\end{array}\right] \\
& B=\left[\sum y_{j}, \sum y_{j} x_{j}, \sum y_{j} x_{j}^{2}, \sum y_{j} x_{j}^{3}\right]^{T}
\end{aligned}
$$

For a resulting function curve $y=f(x)$, the first two derivatives of $f$ at point pi with the new translated coordinates $(0,0)$ are given by

$$
\begin{aligned}
& \mathrm{f}^{\prime}(0)=\mathrm{b} \\
& \mathrm{f}^{\prime \prime}(0)=2 \mathrm{c}
\end{aligned}
$$

$f^{\prime}(0)=b$ gives the slope of the tangent line to the fitted curve at $p_{i}$. Thus, the slope of the normal line at $\mathrm{p}_{1}$ is $-1 / \mathrm{b}$. The normal line at $\mathrm{p}_{1}$ with its original coordinates $\left(\mathrm{x}_{\mathrm{i}}, \mathrm{y}_{\mathrm{i}}\right)$ has the following equation:

$$
y=(-1 / b) x+\left(y_{i}+x_{i} / b\right)
$$

For a boundary point $\mathrm{p}_{\mathrm{i}}$ on the circle, the center of the circle must lie on the normal given by eq. (2). We can further determine the interval of the normal line that the center may lie on by examining the concavity of the circular arc. A curve $\mathrm{f}$ is concave upward if every tangent to the curve of $f$ intersects the curve only at the point of tangency and otherwise lies entirely below the curve of $\mathrm{f}$. A curve $\mathrm{f}$ is concave downward if every tangent lies above the curve of $\mathrm{f}$. Therefore, a curve $\mathrm{f}$ is concave upward if $\mathrm{f}^{\prime \prime}(\mathrm{x})>0$, or $\mathrm{f}$ is concave downward if $\mathrm{f}^{\prime \prime}(\mathrm{x})<0$ for all $\mathrm{x}$ on an open interval. 
Let $\mathrm{y}_{\min }$ and $\mathrm{y}_{\max }$ denote the lower bound and the upper bound, respectively, of the $\mathrm{y}$ coordinate of a circle center. For an image of size $\mathrm{NxN}$, we may have $\mathrm{y}_{\min }=0$ and $\mathrm{y}_{\max }=\mathrm{N}-1$. Consider a concave upward arc as shown in Figure 1(a). The circle center must lie along the half-line of the normal above the arc. Therefore, for a given point $\mathrm{p}_{\mathrm{i}}=\left(\mathrm{x}_{\mathrm{i}}, \mathrm{y}_{\mathrm{i}}\right)$ on the concave upward arc, the possible y-coordinate values of the circle center must be larger than $y_{i}$, i.e., the $y$ coordinate of the circle center is on the interval $\left[y_{i}, y_{\max }\right]$. Similarly, consider a concave downward arc as shown in Figure 1(b). The circle center must lie along the half-line of the normal below the arc. For a given point $\mathrm{p}_{\mathrm{i}}=\left(\mathrm{x}_{\mathrm{i}}, \mathrm{y}_{\mathrm{i}}\right)$ on the concave downward arc, the possible y-coordinate values of the center must be smaller than $y_{i}$, i.e., the y coordinate of the circle center is on the interval $\left[y_{\min }, y_{i}\right]$. For a given $y$-coordinate value $y$, the corresponding $x$-coordinate value $\mathrm{x}$ on a normal line at point $\mathrm{p}_{\mathrm{i}}=\left(\mathrm{x}_{\mathrm{i}}, \mathrm{y}_{\mathrm{i}}\right)$ can be computed by rewriting eq. (2) as follows:

$$
x=-b \cdot y+b \cdot y_{i}+x_{i}
$$

$$
\begin{aligned}
& \text { Or, } \\
& \qquad x=-f^{\prime}(0) \cdot y+f^{\prime}(0) \cdot y_{i}+x_{i}
\end{aligned}
$$

The voting process for detecting the circle centers is proceeded as follows:

Let $P=\left\{p_{i}=\left(x_{i}, y_{i}\right), i=1,2, \ldots, n\right\}$ be the sequence of $n$ boundary points of a part in the image.

For every boundary point $\mathrm{p}_{\mathrm{i}}=\left(\mathrm{x}_{\mathrm{i}}, \mathrm{y}_{\mathrm{i}}\right), \mathrm{i}=1,2, \ldots, \mathrm{n}$

Estimate the cubic polynomial function $f(x)$ at point $p_{i}=\left(x_{i}, y_{i}\right)$ using eq. (1)

$$
\text { For } y= \begin{cases}y_{i,} y_{i+1}, \varphi, y_{\max } & \text { if } f^{\prime \prime}(0)>0 \\ y_{\text {min }}, y_{\text {min }+1}, \varphi^{\prime}, y_{i} & \text { if } f^{\prime \prime}(0)<0 \\ y_{\text {min }}, y_{\text {min }+1}, \varphi^{\prime}, y_{\max } & \text { if } f^{\prime \prime}(0)=0\end{cases}
$$

$$
\text { Compute } x=-f^{\prime}(0) \cdot y+f^{\prime}(0) \cdot y_{i}+x_{i}
$$




$$
\text { Let } A_{c}(x, y)=A_{c}(x, y)+1
$$

\section{End}

\section{End}

This concludes the first stage of the voting process for circle center detection. The accumulator elements $\mathrm{A}_{\mathrm{c}}(\mathrm{x}, \mathrm{y})$ containing sufficiently large numbers of votes against a predefined threshold $\mathrm{T}_{\mathrm{d}}$ are registered as the candidates of circle centers. These resulting centers are the input to the second stage of the voting process for radii detection.

Let $\mathrm{C}=\left\{\mathrm{c}_{\mathrm{j}}=\left(\overline{\mathrm{x}}_{\mathrm{j}}, \overline{\mathrm{y}}_{\mathrm{j}}\right), \mathrm{j}=1,2, \ldots, \mathrm{m}\right\}$ denote a set of $\mathrm{m}$ centers of circular arcs detected in the first stage of the voting process. The voting process for detecting the radii of circles is given as follows:

For every circle center $c_{j}=\left(\bar{x}_{j}, \bar{y}_{j}\right), j=1,2, \ldots, m$

Clean $A_{R}(r)$ for all $r$

For every boundary point $\mathrm{p}_{\mathrm{i}}=\left(\mathrm{x}_{\mathrm{i}}, \mathrm{y}_{\mathrm{i}}\right), \mathrm{i}=1,2, \ldots, \mathrm{n}$

$$
\text { Compute } r=\left[\left(x_{i}-\bar{x}_{j}\right)^{2}+\left(y_{i}-\bar{y}_{j}\right)^{2}\right]^{1 / 2}
$$

Let $A_{R}(r)=A_{R}(r)+1$

\section{End}

If $A_{R}(r)$ is greater than the predefined threshold $T_{d}, r$ is the radius of the circle centered at $\mathrm{c}_{\mathrm{j}}$.

\section{End}

This concludes the second stage of the voting process for radii detection. Note that a given center $c_{j}$ may have many radii if it is the common center of concentric circles. 
The two-dimensional accumulator array $\mathrm{A}_{\mathrm{c}}(\mathrm{x}, \mathrm{y})$ used in the first stage can be eliminated as soon as the circle centers are recorded in the set $\mathrm{C}$. This released memory can be used in the second stage for the one-dimensional accumulator array $A_{R}(r)$. Therefore, the searching of a circle whose center is within an image of size of $\mathrm{N} \mathrm{x} \mathrm{N}$, and whose radius is less than or equal to $\mathrm{N}$ (or is specified with the precision up to $\mathrm{N}^{2}$ quantization increments), only requires a storage space of $\mathrm{N}^{2}$. The time complexity of the proposed two-stage algorithm in the worst case is given by

$$
\mathrm{N} \cdot \mathrm{n}+\mathrm{m} \cdot \mathrm{n}
$$

where $\mathrm{N}$ is the image size in the $\mathrm{y}$ dimension, $\mathrm{n}$ is the number of boundary points in the image, and $\mathrm{m}$ is the number of circle centers detected in the first stage. Since the concavity information of circular arcs is utilized, the number of possible $\mathrm{y}$-coordinate values is less than $\mathrm{N} . \mathrm{N} \cdot \mathrm{n}$ and $\mathrm{m} \cdot \mathrm{n}$ specify the time requirements for the first stage and the second stage of the voting process, respectively.

\section{EXPERIMENTAL RESULTS}

Figures 2(a) and 2(b) demonstrate a circle with a full boundary (2 $\pi$ ), and a half of the boundary (п ), respectively. The circles with partial distortion on the circle boundaries are also displayed in the figures. The bright spots in the images indicate the common intersection points of the normal lines, and are the locations of circle centers. The occlusion of objects does not affect the significance of the center location. The more boundary points available on a circle, the brighter (larger vote counts) the spots and more concentrated the intersection points to the ideal center locations. 
In the experiments, the region of support $\mathrm{k}=8$ is used for curve fitting. Twenty planar, dark circular objects with the radii ranging from 25 to 60 pixels are tested using the proposed algorithm. The resulting mean radius deviation is within 1.2 pixels. In order to evaluate the performance of the proposed method, ten synthetic circles with two radii and various arc angles are also analyzed so that the estimated circle centers and radii can be compared with the ideal parameter values. The ten circles of study are generated in an image of size $512 \times 480$ pixels. The digital boundaries of circles are created such that

$$
\max \left\{\left|\mathrm{x}_{\mathrm{i}}-\mathrm{x}_{\mathrm{i}-1}\right|,\left|\mathrm{y}_{\mathrm{i}}-\mathrm{y}_{\mathrm{i}-1}\right|\right\}=1, \forall \mathrm{i}
$$

Here $\left(x_{i}, y_{i}\right)$ are the coordinates of boundary points generated from the equation of a circle with the radius $r$ and the center at $(254,243)$ approximating to the center of the image, i. e.,

$$
\left(\mathrm{x}_{\mathrm{i}}-254\right)^{2}+\left(\mathrm{y}_{\mathrm{i}}-243\right)^{2}=\mathrm{r}^{2}, \quad \forall \mathrm{i}
$$

Also, $\left(\mathrm{x}_{\mathrm{i}}, \mathrm{y}_{\mathrm{i}}\right)$ are truncated to their nearest integers to represent the coordinates in image space. Table 1 shows the performance of the estimated parameters of two synthetic circles, one with radius 100 pixels and the other one with radius 50 pixels. Each circle is tested under various arc angles (arc lengths), ranging from $2 \pi(100 \%), 3 \pi / 2(75 \%), \pi(50 \%), \pi / 2(25 \%)$ to $/ 4$ (12.5\%). Each dimension of the parameter space is quantized to resolve 1 pixel changes. The errors of the estimated parameters, the $\mathrm{x}$ and $\mathrm{y}$ coordinates of the centers and the radii, are within 1 pixel for both circles with the arc angles larger than or equal to $\pi(50 \%)$. All estimated errors of the circle parameters for the small circle of radius 50 are within 1 pixel even though the arc angle is as small asm /4 (12.5\%). However, the estimated parameter errors are more significant (up to 5 pixels) for the large circle of radius 100 with the arc angles smaller thanr (50\%). This is due to the impact of the angular deviation of the estimated normal for the large circle. The accuracy of the estimated center location is determined by the precision of the normal slope derived from the cubic 
polynomial fitting method. If an estimated normal has angular deviation $\Delta \phi$ with respect to the ideal normal, then the displacement from the ideal center to the estimated normal line is given by $\mathrm{r} \cdot \sin \Delta \phi$, where $\mathrm{r}$ is the ideal radius (see Figure 3). Therefore, the estimated parameter errors of the large circle is larger than those of the small circle. Experimental results have shown that the mean angular deviation of a normal line using the cubic polynomial fitting method is within $2^{\circ}$.

The applications of the proposed method to the detection of circular arcs of industrial parts are demonstrated in Figures 4 and 5. Figures 4(a) and 4(c) show the real images of two oil seals, one with peripheral defect, and the other one with burrs, respectively. The estimated circles of these two oil seals are presented in Figures $4(\mathrm{~b})$ and $4(\mathrm{~d})$. Note that the extruding portions (peripheral defect and burrs) of the oil seals can be easily detected in the image since they are located outside the estimated circles. The crosses "+" shown in the figures mark the locations of the estimated circle centers. The estimated circles coincide with the circular portions of the boundaries of the parts, as seen in Figures 4(b) and 4(d). Figure 5(a) illustrates the binary image of a plate cam, and Figure 5(b) shows two detected circular arcs on the contour of the cam. Figures 5(c) and 5(e) show the profiles of a brake shoe and a friction plate, respectively. The estimated circles for the outermost circular arcs of these two mechanical parts are illustrated in Figures 5(d) and 5(f).

\section{CONCLUSION}

In this paper, we have presented a two-stage Hough transform method for detecting circular parts with partial occlusion and parts with circular arcs on the contours. The resulting circle of the proposed method can be further extended to detect the peripheral breakdown, defects or burrs of a circular parts by evaluating the variation of radial distances between the estimated center and boundary points. 
The proposed method utilizes the directional information of the normal at each boundary point on the circle to increase the computational efficiency and reduce storage requirements. A cubic polynomial curve fitting is employed to estimate the slope angle of a normal and determine the concavity of the fitted curve. The first stage of the voting process first determines the line segment of the normal at each boundary point and accumulates the occurrences of all coordinates on the line segment in a 2-D accumulator. The second stage uses a 1-D accumulator to store the number of individual distances between each estimated circle center and all boundary points. For an image of size $\mathrm{N} x \mathrm{~N}$, this proposed method only requires an accumulator of size $\mathrm{N}^{2}$ and the time complexity is bounded by $(\mathrm{N}+\mathrm{m}) \mathrm{n}$, where $\mathrm{m}$ is the number of circle centers detected in the first stage and $\mathrm{n}$ is the number of boundary points in the image. 


\section{References}

1. U. M. Landau. Estimation of a circular arc center and its radius Computer Vision, Graphics, and Image Processing, 38, 317-326 (1987).

2. S. M. Thomas and Y. T. Chan. A simple approach for the estimation of circular arc center and its radius. Computer Vision, Graphics, and Image Processing, 45, 362-370 (1989).

3. R. Takiyama and N. Ono. A least square error estimation of the center and radii of concentric arcs. Pattern Recognition Letters, 10, 237-242 (1989).

4. P. V. C. Hough. A method and means for recognizing complex pattern. U. S. Patent 3069654 (1962).

5. R. O. Duda and P. E. Hart. Uses of the Hough transform to detect lines and curves in pictures. Commun. ACM, 15, 11-15 (1972).

6. R. S. Conker. A dual plane variation of the Hough transform for detecting non-concentric circles of different radii. ibid., 43, 115-132 (1988).

7. R. K. K. Yip, P. K. S. Tam and D. N. K. Leun. Modification of Hough transform for circles and ellipses detection using a 2-dimensional array. Pattern Recognition, 25,1007-1022 (1992).

8. M. C. Fairhurst. Computer Vision for Robotic Systems: An Introduction. Prentice Hall, Englewood Cliffs, N.J. (1988).

9. C. -H. Teh and R. C. Chin. On the detection of dominant point on digital curves. IEEE Trans. Pattern Anal. Mach. Intell., 8, 859-872 (1989). 